\title{
Spicilegium Salicum Japonensium novarum aut imperfecte cognitarum
}

$\mathrm{By}$

\section{G. Koidzumi}

The most important contribution recently made to our knowledge on the Japanese Salices has been Von Sfemen's Monograph, in which, besides four imperfectly known and seven doubtful ones, thirty-three species, with several varieties and forms, are recognized as occurring in the Archipelago. After that, LÉvEIILE and VANIOT have described three from the collections of FAURIE and Kinashi ; but their Salix Kinashii is identical with Salix triandra. My Salix hondoensis is a distinct species having the characteristic leaves and the elongated style; I think it is convenient to put this, with Salix koreensis Anders., into the new section of Heteradeniæ-DiandræDolichostylæ. However, the peculiar colouring of hair of the catkin in Salix vulpina, is not so important a character as to establish a special section, so I reject the section Vulpinaz of SEEMEN, including it in the section Hastatæ. It seems to me that, Salix Sieboldiana, S. Buergeriana, S. Saidaiana, and S. Harmsiana are but forms or varieties of one species, and at the same time, SEEmen's Salix Matsumuraei and S. daiseniensis had better be considered as varieties of the $S$. vulpina. In this paper three additional species have been descrided; thus bringing the number of species of distinctly known Salices to thirity-two.

1. Salix (Pentandræ) glandulosa Seem. in ENGr. Bot. Jahrb. XXI. (1896), Beibl. LIII. 55 ; Sal. Jap. (1903) 22 ;-LEvL. et VNT. in Bull. Akad. Int. Geogr. Bot. XIII. (1904), 208 ; XVI. (1906) 144, 148 ;-Diels in Engl. Bot. Jahrb. XXIX. 276 ;-NakaI Fl. Kor. II. 214 ;-ShiraI in Tok. Bot. Mag. XVII. (1903) 223, t. 4 . 
Nom. JaP. Akame-yanagi.

Distr. Japonia australis, Korea, China centralis.

var. Warburgi (SEEM.)

S. Warburgi SeEm. in ENGL. 1. c. XXIII. (1897), Beibl. LIII. 43 ;-Forr. et Hemsl. Jour. Linn. Soc. XXVI. 534;Matsum. et Hayat. Enum. P1. Formos. 395.

Differt a typo, foliis utrinque secus costas densius, subtus ramulis hornotinisque laxius pubescentibus; foliis nascentibus saepe densissime brunneo-pubescentibus; bracteolis fl. $\hat{o}$. extus glabris.

Nom. Jap. Takasago-Akameyanagi.

Hab. Formosa.

2. Salix (Subfragiles) eriocarpa Fr. et Sav. Enum. Pl. Jap. I. (1875), 459 ; II. (1879), 503 ;-SEEM. Sal. Jap. (1903) 76.

S. dolichostyla SeEm. in EngL. Bot. Jahrb. XXX. (1902), Beibl. 67, (1901) 39 ; Sal. Jap. 26 ;-LEvL. et VNT. in Bull. Akad. Int. Geogr. Soc. Bot. (1904) 208, (1906) 146, 148.

S. dolichostyla var. hirosakense LeVL. et VNT. in FEdD. Repert. Nov. Spec. III. 22.

Nom. Jap. Kogome-Yanagi.

Hab. Aomori, Hirosaki, Nikko, Hitatsi, Idsu, Suruga, Bittsiu ; Tosa.

"Arbor ramulis novellis pubescentibus; folia lanceolata in petiolo 3-6mill. longo attenuata, elevato-punctata, subtus pallida, demum glabrescentia: amenta $q$, pedunculo brevi (vix 1 $\mathrm{cm}$ ) cum 2-3 foliis minutis ; squamæ concolores : capsulæ sessiles, densissime albo-tomentosae : stylus elongatus!" (FR. et SAv. II. 503.)

Folia lanceolata utrinque attenuata, elevato-punctata, iis Salicis koreensis Anders. simillima!” (Von SFemen, Sal. Jap. p. 76.)

\section{Salix (Subalbæ*) hondœnsis sp. nov.}

* Heteradeniae, Diandrae, Dolichostylae Sect. Subalbae nov. sect., Bracte: concoloria. Fl. S: stamina 2, filamentis puberulis, glandulis 2; 우 ovaria sessilia, stylo elongato, stigmate revoluto-bilobato glanduli 1. Arbor foliis serratis stipulatis. 
S. alba Thg. Fl. Jap. (1784, p. 25, (non Linn.)

Haec species sine dubio $S$. jessoensi Seem. valde affinis, differt floribus amenti brevioris laxioribus; stylis elongatis, stigmatis laciniis leviter emarginato-lobulatis ; glandulis posterioribus ovatis obtusis.

Arbor; ramulis hornotinis velutino-tomentosis ; ramis vetustioribus glabris. Folia lanceolata acuminata serrulata, rarius anguste oblonga acuta, supra atro-viridia minute papillosa, subtus glauca mox glabrescentia; petiolis $2-5 \mathrm{~mm}$. longis velutinis ; stipulis oblique ovatis acuminatis; nervis secundariis arcuato-ascendentibus. Amenta circ. $2 \mathrm{~cm}$. longa, foliis coætanea; rhachibus cano-barbatis; bracteis late ellipticis vel ovato-ellipticis, apice rotundatis raro emarginatis, utraque basi villosis. Fl. $\hat{0}$ : stamina 2 ; filamentis infra medium dense puberulentibus; glandulis 2, anterioribus ovatis obtusis, posterioribus linearibus. Fl. $\delta$ : ovaria lanceolato-ovoidea tomentosa; stylis linearibus glabris; stigmatibus revolutis leviter lobulatis; glandulis 1 ovatis apice obtusissimis.

Nom. Jap. Siro-yanagi.

Hab. Yezo : Sapporo. Nippon: Aomori ; Yonezawa, Adsumasan; Kuragawahara (Iwasiro); Siobara ; Tōkyō, Yokosuka ; Hakone.

4. Salix (Subalbæ) koreensis Anders. in DC. Prodr. XVI. 2, (1868) 217.

Arbor glabra; foliis lanceolatis utrinque attenuatis, serrulatis, glabris, subtus glaucis, sub lente punctulatis; amentis sessilibus foliis praecocioribus; $f$. $t$ : ovaria ovoidea albotomentosa, stylis elongatis, stigmatibus emarginatis, glandulis late ovatis; fl. $\hat{\delta}$ : stamina 2 , filamentis basi barbatis, glandulis 2 ; bracteolis ovatis obtusis concoloribus pilosis.

HAB. Korea.

5. Salix (Hastatæ ${ }^{* *}$ ) vulpina Anders. in Gray, Pl. of Jap. in Mem. Amer. Acad. Arts. Sci. VI, (1857) p. 452 ;-MiQ. Prol.

** Sect. Hastatae Anders. in DC. Prodr. XVI. 2. (1868) 251.=Sect. Vulpincle SEEM. Sal. Jap. (1903) 17 ! 
Fl. Jap. 217 ;-Fr. et SAv. Enum. Pl. Jap. I. 461 ;-KaWAK. Tok. Bot. Mag. X. (1896) 50 ;-Tokub. ibid. 123 ;-Levl. Bull. Acad. Int. Geogr. Bot. (1904) 208 ; (1906) 144 ;-SeEm. Sal. Jap. 37

S. Miqueli var. vulpina Anders. in DC. Prodr. XVI. 2, p. 256

S. vulpina var. discolor SeEm. 1. c. 40.

S. Shiraii var. vulcanica LEvi. 1. c. (1904) 209.

Nom. JAP. Kitsune-yanagi.

HaB. Yezo et Hontō

« typica $\mathrm{m}$.

Ovaria glabra, stigmate rarius bilobulato; bracteis brunneotomentosis.

Hab. Yezo: Osima; Nippon: Mutsu, Rikutsiu, Uzen, Iwasiro, Simozuke, Hitatsi, Izumi.

$\beta$ Matsumuraei (SEem.)

S. Matsumuraei Seem. 1. c. 71 ;-Ievl. 1.c. (1906) 144.

Ovaria stipesque puberula, stigmate lobulato ; bractea albo vel brunneo-tomentosa; capsulis puberulis.

$\mathrm{H}_{\mathrm{AB}}$. Hontō: Gassan; Simizutōge ; Oyamada (Hitatsi).

$\gamma$ nikkoensis $\mathrm{m}$.

Ovaria subsessilia, glabra; stigmate obscuriter bilobulato: bracteis albo-tomentosis, florum $q$ suborbicularibus, $\hat{\delta}$ obovatoellipticis.

Hab. Nikko.

$\delta$ daiseniensis (SEEM.)

S. daiseniensis SeEM. 1. c. 65.

Stamina 2 rarius 1 , filamentis interdum basi vix connatis ; ovaria stipesque puberulento-tomentosa.

Hab : Yezo : Rebunziri ; Nippon : Daisen (Hōki)

$\varepsilon$ pubescens $\mathrm{m}$.

Folia adulta utrinque secus costas pubescentia

HAB. Hontō australis.

$\zeta$ coriacea $\mathrm{m}$.

Folia rigide coriacea.

Hab. Yezo: Prov. Osima. Honto: Prov. Mutsu.

6. Salix (Hastatæ) japonica Thg. Fl. Jap. (1784) 24; Icon. Pl. Jap. Dec. IV. t. 1 ;-Gray in Perry Exped. Jap. 2. p. 319 ;- 
Anders. in A. Gray, Bot. Jap. App. p. 450 ;-MiQ. Prol. Fl. Jap. $212 ;-F_{R}$. et SAv. Enum. Pl. Jap. I. 459, II. 503 ;-SEEM. Sal. Jap. 43 ;-C. K. Schn. I11. Handb. Laubh. I. 50.

S. japonica var. nipponensis LEvL. in Bull. Acad. Int. Geogr. Bot. (1904) 209.

S. japonica var. pygmæa Fr. et SAv. Enum. Pl. Jap. II. 503.

S. padifolia Anders. in A. Gray, Bot. Jap. 1. c. 451, (1858 -59);-DC. Prodr. XVI. 2. p. 255 ;-Tokub. Tok. Bot. Mag. X. p. 123 .

S. Oldhami MiQ. Ann. Mus. Bot. Lugd. Bat. III, (1867) 25.

S. padifolia var. viridula AnDERs. in DC. 1. c. 256.

S. viridula ANDERs. in Gray, 1. c. 451.

S. babylonica var. japonica Anders. in DC. 1. c. 213.

Nom. Jap. Siba-yanagi.

Нав. Yezo, Hontō, et Sikok.

f. 1 typica $\mathrm{m}$.

Folia lanceolata, caudato-acuminata.

f. 2 padifolia $m$.

S. japonica var. padifolia Seem. 1. c. 45.

Folia oblonga acuta vel subito acuta.

\section{f. 3 Oldhami $\mathrm{m}$.}

S. japonica văr. Oldhami Fr. et SAv. Fnum. Pl. Jap. I. (1875), 459.

Folia oblonga acuminata.

7. Salix (Phyllicifolia) Reinii Fr. et Sav. Enum. Pl. Jap. I. (1875) 459, II (1879) 503 ;-Seem. Sal. Jap. 41 ;-LevL. in Bull. Acad. Int. Geogr. Bot. (1904) 208, (1906) 143.

S. glabra Fr. et Sav. 1. c. II. 503 ;-Kawak. Tok. Bot. Mag. X. (1896) 50 ;-Tokub. ibid. 123. (non Scop.)

A S. glabra Scop. cui affinis, foliis juvenilibus utrinque pubescentibus; bracteis florum $f$ intus pubescentibus demum pilosis ; glandulis ovoideo-lanceolatis stipiti ovarii subæquilongis ; ovario stipesque puberulente recedit.

var. cyclophylloides $\mathrm{m}$.

Foliis obovalibus vel orbicularibus rarius late ellipticis obtusis 
juvenilibus utrinque sæpe ferrugineo-tomentosis ; amentis fructiferis multo brevioribus circ. $15 \mathrm{~mm}$ longis ;

Nom. Jap. Maruba-Mineyanagi.

Hab. Hontō: Iwatesan et Hayachinesan (Prov. Rikutsiu.)

8. Salix (Purpureæ) purpurea L. Sp. Pl. (1753) p. 1017.Anders. in DC. Prodr. XVI. 2. p. $306 ;-$ C. K. Schn. Ill. Handb. Laubh. I. 68 ;-SEem. Sal. Jap. 54.

Subsp. eupurpurea Schn. var. sericea W. Kocн, Syn. 644 ;-Schn. 1. c. 69.

S. purpurea $\beta$ sericea SEEM. 1. c. 56.

Nom. Jap. Kawa-yanagi

DisT. in regionibus borealis Gerontogæa.

Subsp. amplexicaulis Borss. Fl. Or. IV. (1879) 1187 ;C. K. Schn. 1. c. 69.

S. amplexicaulis Bory et Chaub. Exp. Sci. Mor. III. 2, (1832) 277 ;-Halacsy, Consp. Fl. Gr. III. 138.

S. multinervis Fr. et Sav. Enum. Pl. Jap. II. 504.

S. purpurea var. multinervis Matsum., Seem. Sal. Jap. 56.

Folia opposita lineari-oblonga sessilia amplexicaulia.

Nom. JaP. Inu-koriyanagi.

HAB. in regionibus borealis Gerontogææ.

var. petiolata $\mathrm{m}$.

Foliis oblanceolato-oblongis vel subpandulaformibus, apice obtusis usque rotundatis basi rotundatis magis minusve petiolatis.

Nom. JaP. Ohba-koriyanagi.

Has. Manshuria

subvar. angustifolia m.

Foliis linearibus vel anguste lanceolatis, 5-8 raro $12 \mathrm{~mm}$ latis.

Nom. Jar. Hosoba-koriyanagi.

Hab. Yezo: Prov. Isikari.

9. Salix (Subviminales) gracilistyla MiQ. Ann. Mus. Bot. Lugd. Bat. III, (1867) 26 ;-Fr. ct SAv. Enum. Pl. Jap, I. 461 ; -Mig. Prol. Fl. Jap. 214 ;-Tokub. Tok. Bot. Mag. X. 124. 
S. Thunbergiana BI. ex Anders. in DC. Prodr. XVI. 2, (1868) 271 ;-Seem. Sal. Jap. 61 :-Leve. in Bull. Acad. Int. Geogr. Bot. (1904) 210 ;-YABE, in Tok. Bot. Mag. (1903) 174 ;-Komaro. Fl. Mansh. II. 30 ;-NaKaI, Fl. Kor. II.

Nom. JAP. Neko-yanagi.

Distr. Japonia, Korea, Manshuria.

10. Salix (Sieboldianæ) Sieboldiana Br. Bijdragen X. (1825) 517 ;-SEem. Sal. Jap. 63.

S. caloptera MiQ. Ann. Mus. Lugd. Bat. III. 26.

? S. brachylepis Fr. et Sav. Enum. P1. Jap. II. 503.

Frutices ; ramulis novellis cinereo-tomentosis ; foliis nascentibus ferrugineo-tomentosis, adultis glabris rigide coriaceis, supra nitide viridibus, subtus glaucinis, argute scrratis, oblongis acutis, nervis secundariis utraque latere circ. 11-15; fl. $\uparrow$ : stamina 2 vel 1 ; filamentis basi puberulis liberis vel infra. medium rarius usque ad extremum apicem connatis; $q$ : ovaria breviter pedicellata tomentosa; bracteis albo-tomentosis stipem superantibus.

NoM. JAP. Iwa-yanagi, Yama-yanagi.

Hab. Japonia australis.

\section{\%. typica m.}

Fl. $\uparrow$ : stamina 2 raro 1 , filamentis liberis vel alte connatis. $\beta$ Buergeriana (Mro.) $\mathrm{m}$.

S. Buergeriana Mig. Ann. Mus. Bot. Lugd. Bat. III. (1867) 28 ;-SeEm. Sal. Jap, 66.

S. Saidæana Seem. 1. c. 68.

S. Harmsiana Seem. 1. c, 73. 80.

Fl. $\hat{\jmath}$ : stamina 1 .

f. 1 genuina $m$.

Amenta præcocia, $t$ usque $9 \mathrm{~cm}$ longa.

f. 2 Saidaeana $\mathrm{m}$.

Amenta coætanea.

f. 3 Harmsiana $m$.

Ovaria brevissime stipitata.

$\gamma$ sikokiana $\mathrm{m}$. 
Folia tenuiora subtus pilis laxe persistentibus; floribus $q$ laxius confertis.

HAB. Sikok: Tsurugisan, Yahazuyama.

11. Salix (Amygdalinæ) amygdalina L. Sp. Pl (1753) p. 1016 ;-Maxim. Prim. Fl. Amur. 242 ;-RgL. Fl. Uss. nr. 435 ;Fr. Schmdt. Reis. Amug, nr. 327 ;-C. K. Schn. Ill. Handb. I. 30 .

S. triandra L. 1. e. 1016 ;-ANDERs. in DC. Prodr. XVI. 2. p. 202 ;-Korsh. Act. Hort. Petr. XII. 390 ;-Hlerder, ibid. XI. 395 ;-Forb. et Hemsl. Jour. Lim. Soc. XXVI. 533 ;-Komarov. Fl. Mansh. II. 30 ;-Levi. Bull. Akd. Int. Geogr. Bot. (1906) $144,148$.

S. nipponica Fr. et SAv. Enum. P1. Jap. II. 502.

S. triandra var. nipponica Seem. Sal. Jap. 27.

S. Kinashii Levi. Bull. Soc. Bot. Fr. LIII. (1905) 141, Bull. Alkd. Int. Geogr. Bot. XVI (1906) 148, 144.

Nom. Jap. Tachi-yanagi.

Distr. in regionibus borcalis Geronatogre.

12. Salix (Viminales) stipularis Sмiтн, Fl. Br. III. (1804) 1069 ;-Kordz. P1. Sachal. Nakah. (1910) 45.

S. opaca Anders., Schmdt. Reis. Sachal. in Mem. Ac. Imp. Sc. St. Petrsbg. ser. VII. tom. 12, (1858) p. 172.

var. sachalinensis (SснмDT.)

S. sachalinensis Fr. SchmdT. 1. c. (1868) p. 173;-Koldz. 1. c. (1910) 45 .

A typo differt, amentis praecocioribus; foliis versus basin anguste lanceolatis.

Nom. Jap. Karafuto-Yanagi.

Distr. Saghalin, Yezo.

13. Salix (Lanatæ) vulpinoides sp. nov.

Haec planta Salices vulpina et futura habitu approximat: ab illis differt foliis subtus adpresse pubescenti-tomentellis, nervis secundariis basilaribus ut in $S$. Sieboldiana parallele rectius marginem attingentibus; petiolis griseo-tomentosis; bracteis el- 
liptico-ovatis obtusis albo-tomentellis majoribus; ovariis tomentosis sessilibus. Ab hac bracteis ellipticis obtusis brevioribus utrinque albo-tomentosis ; amentis longioribus angustioribusque.

Arbuscula ; ramulis novellis sordide cinereo-tomentosis usque pubescentibus; ramis vetustioribus glabris cortice fusco vel nigrescente ruguloso non nitente vel ex luteo-fusco nitente obductis; gemmis axialibus parvulis glabris. Folia nascentia utrinque cinerco vel brunneo-tomentosa, adulta tenuiter chartacea vel membranacea, supra opaca atro-viridia laxius pilosa, præsertim ad venas cinereo-tomentella, subtus glaucina vel pallide grisea adpresse sericeo-pubescentia; nervis secundariis utraque latere 5-9 arcuato-ascendentibus sed basilaribus rectiusculis, utraque pagina vix prominulis cinereo-tomentosis, unde albolineata videntur; oblonga vel elliptica rarius obovato-elliptica, acuta, basi obtusa usque rotundata rarius subcuneato-angustatá ; margine crenato-serrata ; petiolis $4-13 \mathrm{~mm}$ longis cinereopubescentibus; stipulis falcato-semiovatis extrorsum denticulatis ; lamina (1.5-) 4-9 (-14) cm longa (0.9-) 2-4 (-4.5) $\mathrm{cm}$ lata. Amenta præcocia sessilia vel breviter pedunculata, $4-5 \mathrm{~cm}$ longa, basi foliis parvis supra glabris subtus sericeo-tomentosis suffulta; bracteis ellipticis apice fusco-coloratis obtusis utrinque villosotomentosis. Fl. $q$ : ovaria ovoideo-lanceolata sessilia vel brevissime stipitata, sericeo-tomentosa; stylis mediocribus stigmate profunde lobato, lacinulis patentibus; glandulis ovoideis parvulis; bracteis minoribus obtusioribus. F1. $\hat{\delta}$ : stamina 2 ; filamentis glabris; glandula ovoidea vel ovoideo-oblonga; bracteis majoribus acutioribus. Amenta matura $8 \mathrm{~cm}$ longa, gracile angustata, breviter pedunculata; pedunculis paucifoliatis rhachibusque cinereo-tomentosis; capsulis densius confertis ovoideolanceolatis subsessilibus, extus puberulento-tomentellis, intus glabris.

Nom. Jap. Oh-nekoyanagi (nov.)

Hab. Hontō: Prov. Sinano, Togakusiyama, prope Nagano; Prov. Kodsuke, Myogisan et Akagisan; Prov. Yechigo, prope Nagaoka. 


\section{Salix (Hastatæ) Nakamurana sp. nov.}

Haec species affinis Salix cyclophyllæ SErm., sed ab ea differt foliis tenuioribus glabris ; ramulis novellis, inflorescentiis pedunculisque glabris; glandulis lineari-lanceolatis ; ovario stipitato, apice non attenuato; stigmate integro. Differt etiam a Salix pyrolæfolia LeDEr. et $S$. hastata $\mathrm{L}$. foliis integris glabris subtus minus glaucis tenuioribusque; glandulis linearilanceolatis; filamentis basi leviter connatis; ovario brevius stipitato; stigmatibus linearibus integris.

Fruticulus glaber; foliis ellipticis vel obovato-ellipticis integris, longe petiolatis ; amentis foliato-pedunculosis coætaneis ; bracteolis ellipticis vel oblongis supra medium dilute fuscatis utrinque sericeo-villosis; glandulis 1 lineari-lanceolatis ; $f$. $q$ : stamina 2, filamentis gracile elongatis glabris basi plus minus connatis; fl. $q$ : ovaria ovoideo-lanceolata glabra breviter stipitata; stylo mediocri, stigmate integro.

Fruticulus ramulis novellis laxe villosis cito glabris, vetustioribus cortice fusco vel fusco-purpureo nitidoque vestitis; trunco subterraneo. Gemmæ oblongæ vel ovoideæ. Folia glabra, sed inferiora ramulorum præsertim subtus ad costas laxius villosa, supra dilute viridia, subtus glaucina, elliptica vel obovato-elliptica, rarius oblonga vel obovato-oblonga, integerrima rarissime obscuriter remote serrata ; apice rotundata vel oblique obtusiuscula, interdum emarginato-truncata, basi acuta obtusa vel subcuneato-acuta; nervis secundariis versus apicem arcuato-attingentibus; lamina usque $4.5 \mathrm{~cm}$ longa, $2.3 \mathrm{~cm}$ lata; petiolis glabris ad $15 \mathrm{~mm}$ longis; stipulis saltem in ramis fertilibus deficientibus. Amenta $2.5-4 \mathrm{~cm}$ longa, foliis coætanea, foliatopedunculata; rhachibus laxius villosis; bracteolis ellipticis vel oblongis apice rotundatis, supra medium fusco-colorat is, utrinque villosis; floribus laxius confertis ; nectariis lineari-lanceolatis. Fl. $\hat{\delta}$ : stamina 2, filamentis glabris gracile elongatis basi leviter connatis. F1. $q$ : ovaria ovoideo-lanceolata, glabra breviter stipitata; stylis mediocribus; stigmate lineari integro. Amenta matura haud elongata.

Nom. Jap. Lenge-iwayanagi (M. NAKamura). 
Hais. Prov. Sinano: Dailengezan, (Leg. M. Nakamura! Aug. 1912.)

I have named this interesting species in memory of $\mathrm{Mr}$. Masao NaKamura, who sent me the valuable materials collected by him.

15. Salix (Sericeæ) arctica Pall. Fl. Ross. II. (1788) 86 ; Ledeb. Fl. Ross. III. 619 ; Anders. in DC. Prodr. XVI. 2, p. 286; Herder in Act. Hort. Petr. XI. 438; C. K. Schn. 1. c. 41. S. Pallasi, Anders. 1. c. 285 ; Ledeb. 1. c. 619.

Nom. Jap. Chisima-yanagi.

Нав. Kurile: Simshu, Urup.

DISTR. regionibus arcticis et subarcticis.

16. Salix (Urbanianæ) cardiophylla TR. et Mey, F1. Ochot. (1856) 77 ; SEEM. Sal. Jap. 77.

Nom. JAP. Tokatsi-yanagi.

Hab. Simizutoge (Yechigo); Senjugahama (Nikko); Osima, Tokatsi.

Distr. Sibiria orientalis et Manshuria. 